\title{
Dependência de recursos e custos de transação: rumo a um modelo convergente
}

\author{
Resource dependence and transaction costs: \\ towards a convergent model
}

\author{
Arleson Eduardo Monte Palma Lopes
}

\begin{abstract}
Resumo: No presente trabalho abordaremos o processo das relações interorganizacionais utilizando em sua análise duas perspectivas: a dependência de recursos e a teoria dos custos de transação. No primeiro momento, o texto apresentará uma revisão bibliográfica sobre os dois construtos teóricos e em seguida será feita uma discussão de como a utilização dessas teorias pode contribuir para a compreensão do fenômeno interorganizacional. Apesar das divergências entre as duas perspectivas, os autores, na revisão bibliográfica feita, concluem que elas são convergentes. Na convergência de suas concepções, discorrem a respeito de as relações organizacionais serem o fator-chave para o funcionamento das empresas. A perspectiva da dependência de recursos sugere a distribuição dos recursos no ambiente e, para obtê-los, as organizações desenvolvem relações organizacionais. Já na perspectiva dos custos de transação, discorrem sobre as relações de trocas econômicas entre as organizações. Portanto, para compreender as relações interorganizacionais é preciso considerar as suposições das perspectivas.
\end{abstract}

Palavras-chave: Dependência de recursos; Custos de transação; Relações interorganizacionais.

\begin{abstract}
The present study addresses the process of inter-organizational relationships from two theoretical perspectives: resource dependence and transaction costs. First, the text presents a bibliographic review on the two theoretical constructs; next, it discusses how the use of these theories can contribute to understanding the interorganizational phenomenon. Despite the divergence between the two perspectives, the authors conclude that they are convergent based on the bibliographic review. The convergence of these conceptions relates to the fact that organizational relations are a key factor for the functioning of companies. The resource dependence perspective suggests that resources are distributed in the environment and that firms develop organizational relationships to obtain them; in contrast, the perspective of transaction costs refers to the relations of economic exchange between organizations. Therefore, it is necessary to consider the assumptions of these two theoretical perspectives to understand inter-organizational relationships.
\end{abstract}

Keywords: Resource dependence; Transaction costs; Inter-organizational relationships.

\section{Introdução}

Nos últimos anos, as teorias dos estudos organizacionais têm caminhado cada vez mais para enxergar as organizações como sistemas abertos. Quando vistas como sistemas abertos, as organizações concebem sua sustentação nas interações que desenvolvem com o ambiente. Assim, as organizações adquirem ao longo desse processo a habilidade de adaptar-se ao ambiente e manipulá-lo (Morgan, 2002; Thomazine \& Bispo, 2014).

Portanto, a alavancagem acirrada pela competição no mercado tem exigido das organizações presteza e flexibilidade, criando estruturas de ambiente menos centralizadas. As organizações acabam dessa forma criando um conjunto de relações e vínculos entre si (Puffal \& Puffal, 2014).
Ao buscarem influenciar seu ambiente, as organizações traçam alianças e ajustes estratégicos que permitem controlar com eficiência os recursos. $\mathrm{O}$ estabelecimento de vínculos interorganizacionais implica diretamente em negociações e concessões, portanto, há perda concernente de liberdade e para isso as organizações desenvolvem estratégias com o intuito de conseguir maior estabilidade e, ao mesmo tempo, controle sobre os recursos que procuram, ao máximo, manter sobre sua tutela (Motta \& Vasconcelos, 2015).

A perspectiva da dependência de recursos parte do posicionamento de que a gestão organizacional age de forma eficiente, buscando alternativas às implicações relacionadas à distribuição de recursos críticos às

${ }^{1}$ Universidade Federal do Pará - UFPA, Rua Três Maria, 115, Vila Nova, CEP 68600-000, Bragança, PA, Brasil, e-mail: arlesonlopes93@gmail.com Recebido em Mar. 16, 2016 - Aceito em Jul. 14, 2016

Suporte financeiro: Nenhum. 
atividades das organizações em determinado ambiente (Rocha et al., 2011). A capacidade organizacional para catalisar os recursos vitais e o gerenciamento interorganizacionais são instrumentos fundamentais nessa perspectiva (Pfeffer, 1972; Rossetto \& Rossetto, 1999).

Assim, segundo Thomazine \& Bispo (2014, p. 2),

[...] as organizações devem realizar relações de troca com outras organizações, elas podem alterar estruturas e comportamentos para adquirir e manter os recursos necessários.

As transações que surgem da relação de trocas entre as organizações envolvem custos, além de controle e poder. A perspectiva dos custos de transação tem como base de análise a importância das organizações para apreciação econômica, tendo em vista que as crescentes falhas que podem surgir no mercado aumentam os custos de transação (Williamson, 1979; Balestrin \& Vargas, 2002; Pereira et al., 2014; Thomazine \& Bispo, 2014).

Desse modo, o ponto de partida deste artigo é um ensaio teórico que busca discutir comparativamente duas abordagens teóricas: dependência de recursos e custos de transação, bem como seu potencial de complementaridade, para explicar o dinamismo das relações interorganizacionais.

Apesar de as duas correntes teóricas adotarem visões divergentes em sua base de análise, na revisão bibliográfica pode-se notar que elas se entrelaçam quando reconhecem os efeitos das relações de trocas que surgem no campo organizacional.

\section{Dependência de recursos}

De modo análogo à teoria dos sistemas abertos e teoria da contingência, a teoria da dependência de recursos parte do pressuposto de que as empresas são dependentes do ambiente quando se trada da obtenção dos recursos necessários à sua sobrevivência e desenvolvimento (Rossetto \& Rossetto, 1999; Bucelli et al., 2014; Motta \& Vasconcelos, 2015).

Segundo Balsini et al. (2005), a teoria da dependência de recursos tem como concepção o fato de as organizações não serem capazes de produzirem todos os recursos que necessitam para sua sobrevivência. Ou seja, nem todas as atividades exercidas pelas organizações são autossuficientes. Adicionalmente, Rossetto \& Rossetto (1999), Andrade \& Amboni (2011) e Veiga et al. (2014) ressaltam que a teoria da dependência de recursos reconhece os efeitos causados pelo ambiente sobre os resultados das estratégias adotadas pelas organizações, mas também admitem a importância do papel gerencial em saber captar recursos necessários para obtenção de uma performance satisfatória.
Assim, para Motta \& Vasconcelos (2015), devido às dificuldades que as organizações têm na captação dos recursos necessários à sua sobrevivência no ambiente, elas traçam alianças estratégicas com outras empresas que têm acesso a esses recursos do quais necessitam para competir no mercado. Nesse contexto, a teoria da dependência de recursos tem como enfoque a complexidade, dinamismo e o nível de riqueza dos ambientes onde as organizações atuam. Para os autores, o nível de controle dos recursos indispensáveis dos quais a empresa depende é um fator crucial na abordagem da dependência de recursos.

Já Cunha (1993) e Medeiros \& Paiva (2012) destacam que a expansão da dependência das organizações em relação aos recursos depende de quatro atributos: (1) a influência dos recursos oferecidos por outra empresa; (2) a forma de distribuição dos recursos no ambiente, isso poderá elevar o grau de dificuldade na sua obtenção; (3) existência ou não de monopólio de determinado grupo de organizações sobre os recursos; (4) existência ou falta de recursos/serviços substitutos. Segundo Pfeffer (1972), as organizações adotam diversas estratégias para o gerenciamento eficiente da interdependência ambiental. Assim, o autor destaca três fatores para o processo de gerenciamento ambiental: as organizações devem minimizar a interdependência simbiótica; reduzir as relações de interdependências comerciais ou competitivas através de estratégias de obtenção de recursos; e diversificar, visando evitar a dependência do ambiente.

Rocha et al. (2011, p. 37) enfatizam que na

Dependência de Recursos, organizações estariam constantemente buscando discricionariedade, entendida aqui como capacidade de ação liberta de regras ou limites impostos e fiscalizados pela sociedade e demais organizações.

Portanto, as organizações necessitam ser mais estratégicas em relação ao uso de seus recursos, tornando mais difícil a imitação desses recursos. Nessa circunstância, as empresas que conseguirem descobrir a maneira mais eficaz de desenvolver determinada atividade em relação a seus concorrentes apresentarão performance superior nesse ramo (Souza, 2011). Segundo Abbade (2010), as estratégias representam para as organizações uma alternativa de minimizar a dependência do ambiente, intercambiar novos recursos, mercados, alcançar economias de escala e obter vantagem competitiva.

Nesse sentido, as organizações são dependentes dos recursos que necessitam e, por sua vez, são dependentes do ambiente onde estão inseridas. Os recursos dos quais as empresas são dependentes são: tecnologia, matéria-prima, recursos financeiros, pessoal, entre outros (Sacomano \& Truzzi, 2002). Segundo Trevisan (2013), o nível de dependência de recursos críticos das empresas acaba influenciando 
nas ações estratégicas e no processo de tomada de decisões das organizações, destacando-se que essas podem ser explicadas de modo particular.

Rossetto \& Rossetto (2005), Cardenas \& Lopes (2006) ressaltam que as organizações devem traçar estratégias no intuito de captar recursos da forma mais eficiente possível. Como em grande parte os recursos são escassos e de difícil imitação e substituição, acabam levando as empresas fazerem alianças estratégicas. Portanto, quando uma empresa necessita de determinado recurso para sua produção ou operação no mercado, existem três maneiras de consegui-lo: produzindo-o, buscando-o no mercado ou conseguindo por meio de alianças estratégicas com organizações que detêm esses recursos. Segundo Motta \& Vasconcelos (2015), as empresas devem desenvolver uma relação de interdependência simbiótica integrante, mas não competitiva ou excludente. A título de exemplificação dessa relação interorganizacional pode ser destacado o entrelaçamento entre fornecedores-clientes, quando output ou produto de uma organização constituir fornecimento de matéria-prima ou input para outra empresa.

Trevisan (2013) corrobora que as organizações desenvolvem, entre suas escolhas estratégicas, formas de atuar perante o ambiente, com intenção de manipulá-lo. Desse modo, as organizações criam demandas de produtos/serviços ou buscam alianças com outras empresas com o intuito de regrar a concorrência.

Em síntese, a teoria da dependência de recursos adota uma visão voluntarista quando trata das estratégias das organizações para enfrentar as contingências impostas pelo ambiente. Os adeptos da teoria da dependência de recursos enfatizam que as organizações buscam captar recursos no ambiente, ressaltando a relação interorganizacional na aquisição desses recursos.

\section{Teoria dos custos de transação}

A teoria econômica dos custos de transação surge a partir dos estudos de Coase e é posteriormente formulada na década de 1940 por Williamson, considerando que as organizações, no oportunismo como base sutil e generalizada da espécie humana, estabelecem relações interorganizacionais. Assim, a transação era o ponto de análise básica da organização e o propósito central da organização econômica se constituía através das relações de trocas (Costa, 2009; Schubert, 2012).

A perspectiva dos custos de transação faz um corte analítico que privilegia a análise eficiente das relações contratuais entre as organizações, surgindo probabilidade do estudo das organizações como "arranjos institucionais" que conduzem as transações por meio de contratos formais (regidos por leis) ou alianças informais (Cunha et al., 2015).
Os custos de transação são necessários ao processo de negociação, monitoramento e controle de trocas entre as organizações e os agentes econômicos envolvidos, bem com as garantias do cumprimento de um contrato (Ilha, 2010; Diniz \& Marconatto, 2011; Motta \& Vasconcelos, 2015). Assim, a perspectiva do custo de transação decorre da importância das organizações para a análise econômica, tendo em vista as crescentes imperfeições que surgem no mercado, impactando no aumento dos custos de transação (Balestrin \& Vargas, 2002; Pereira et al., 2014).

Os fatores que desenvolvem os construtos da economia dos custos de transação são: oportunismo como perspectiva central no custo de transação, o oportunismo nas atividades econômicas que envolvem investimentos em atividades físicas e humanas e o processo eficiente das informações, que é um dos conceitos importantes para mensuração dos custos de transação (Williamson, 1979).

Nesse sentido, as organizações precisam estabelecer estratégias para se prevenir do oportunismo dos seus parceiros comerciais, conforme exposto por Viana \& Añez (2008, p. 68) na abordagem a seguir:

As organizações são vistas como a resposta aos ambientes incertos, encontrando-se incluídos nesses ambientes os parceiros comerciais cuja confiabilidade é desconhecida e que podem agir de modo oportunista. Posicionar as transações sob a hierarquia da organização permite o monitoramento do comportamento por meio da supervisão direta, auditoria e outros mecanismos de controle.

\section{Lopes (2004, p. 10) afirma que}

[...] o mercado é apontado como a estrutura clássica de governança, no qual compradores e vendedores anônimos (faceless) encontram-se para um instante de troca de bens padronizados, na perspectiva do equilíbrio de preços.

A teoria dos custos de transação é crucial para o processo de tomada de decisão, uma vez que as instituições são permeáveis, tanto internamente quanto externamente, pelas relações de trocas entre os agentes econômicos, sejam eles indivíduos ou firmas. Em um ambiente em que se exige um grau de especialização forte e uma divisão do trabalho racional no qual a transação é o ponto central, a redução da transação incentiva à cooperação e atenua o oportunismo, aumentando os mecanismos de produção de forma eficiente (Schlabitz, 2008).

Os construtos analisados pela teoria econômica dos custos de transação são as especificidades dos ativos envolvidos na transação, o grau de frequência em que ocorrem as transações e o nível de incerteza dos contratos. Todavia, a abordagem teórica considera, por outro lado, aspectos relacionados ao comportamento humano: racionalidade limitada e 
oportunismo. A incerteza e o nível de competitividade podem variar de acordo com as nuanças do mercado (Gary \& Spencer, 2000; Arbage, 2004; Silva, 2006; Schubert, 2012).

As organizações devem traçar um plano de contingência visando prevenirem-se com relação ao oportunismo e às incertezas, minimizando os riscos e, consequentemente, os custos de transação. Portanto, são utilizadas algumas ferramentas: contratos, garantias, seguros e preços mais elevados (Cavalcanti et al., 2002).

Assim, as empresas, buscando se prevenir do oportunismo, redigem contratos bem detalhados, proporcionando maiores garantias e previsibilidade. Contudo, por mais que os contratos sejam bem detalhados, jamais serão completos e preverão todas as contingências que podem surgir em ambiente com constante complexidade (Cavalcanti et al., 2002). Para Kato \& Margarido (2000), as contingências que não fazem parte do ex-ante adicionam custos ex-post, fazendo com que os agentes econômicos envolvidos tenham que renegociar os contratos quando os fatores contingenciais se manifestam.

Quando as organizações optam por verticalizar sua cadeia produtiva, não o fazem somente por compreender que os custos de transação do mercado serão menores, mas por acreditarem em um grau de reconhecimento proeminente (Santos et al., 2014).

Em síntese, a teoria econômica dos custos de transação destaca que agentes econômicos e organizações, em ambientes complexos e dinâmicos, buscam manipular as mudanças e influenciar o curso delas por meio do mecanismo de troca (Motta \& Vasconcelos, 2015).

\section{Análise comparativa das teorias dependência de recursos e custos de transação}

As contribuições teóricas que as duas abordagens têm proporcionado aos estudos organizacionais são bastante relevantes, porém, devido à dinâmica mercadológica que as organizações enfrentam para se sustentarem no mercado; utilizar apenas uma abordagem para analisar as diversas facetas que envolvem o campo organizacional não nos daria as evidências necessárias à compreensão do dinamismo mercadológico. Assim, as proposições teóricas feitas são analisadas a partir de uma perspectiva comparativa, identificando os atributos divergentes e convergentes entre elas, as quais podem ser utilizadas como modelo complementar no processo organizacional.

A correlação entre a teoria da dependência de recursos e a teoria dos custos de transação destaca que quanto maior for o grau de dependência de uma organização dos recursos ofertados por outra, maior será o controle que essa organização irá exercer sobre a empresa dependente dos recursos, com o intuito de minimizar a incerteza e o nível de dependência. Assim, quanto maior for o grau de controle que uma organização procurará exercer sobre outra, para reduzir a incerteza, maiores serão os custos de transação envolvidos na operação (Dimaggio \& Powell, 2005; Camilo et al., 2012; Thomazine \& Bispo, 2014; Motta \& Vasconcelos, 2015).

Diante do exposto, Medeiros \& Paiva (2012, p. 6) explicam o grau de vulnerabilidade das organizações no ambiente e as estratégias que as organizações adotam para minimizar essa dependência.

\begin{abstract}
Quanto maior for a dependência de determinado recursos por parte de uma organização, maior será sua vulnerabilidade perante o mercado. Assim sendo, a fim de diminuir tal vulnerabilidade, a empresa aliar-se a outros agentes, o que lhe confere maior acesso aos recursos de que necessita, minorando sua dependência com relação ao ambiente.
\end{abstract}

O processo de estrutura do campo organizacional se fundamenta de diversos atributos, os quais incluem elementos como: aumento das interações entre as organizações; advento de arquitetura de dominação e coalizações interorganizacionais; a desenvoltura na carga de informação com a qual as organizações devem lidar; e a ampliação de uma conscientização mútua entre as organizações que atuam em um mesmo nicho de mercado (Dimaggio \& Powell, 2005; Gonçalves; 2008).

De modo análogo, quando a organização corre o risco de ficar sem recursos dos quais necessita, irá estabelecer um acordo formal com outra organização, da qual depende. Essa relação de dependência dos recursos aumentará os custos de transação (Motta \& Vasconcelos, 2015).

As organizações desenvolvem relações de troca com outras instituições, podendo modificar estruturas e comportamentos para catalisar e manter os recursos. A relação interorganizacional entre as organizações envolve custos e uma perspectiva de poder e controle. Portanto, a ênfase dada ao relacionamento organizacional tem aspectos unificados aos custos da gestão de contratos e estruturas de governança, é o que desenvolveu a teoria dos custos de transação (Thomazine \& Bispo, 2014).

As organizações passam, assim, a serem controladas através das relações de interdependência com seu ambiente. As relações de interdependência entre as organizações estão associadas aos padrões de troca de recursos e atividades da organização, nos quais essas relações explicam de modo parcial a rentabilidade das empresas (Pfeffer, 1972; Jacobs, 1974; Viana \& Añez, 2008).

Bataglia et al. (2009) afirmam que quanto mais incerto se estruturar o ambiente, maior será o grau de vulnerabilidade das organizações na constituição de trocas e, consequentemente, maiores esforços serão 
direcionados para controlar a incerteza através do estabelecimento de contratos. Os contratos podem ser estruturados em explícitos e formais, assim como os contratos de distribuição exaustiva, ou implícitos, como no caso de formação de alianças estratégicas informais. Esse mecanismo provoca a crescente necessidade de obter informações, base de negociação e desenvoltura de controle, aumentando de modo substancial os custos de transação.

Portanto, quando as organizações desenvolvem alianças estratégicas, geram uma relação de interdependência que visa obter os recursos do quais necessitam ou a formulação de contratos com outras organizações, buscando se prevenir do oportunismo.

As alianças estratégicas envolvem acordos voluntários com o intuito de cooperação interorganizacional, na maioria das vezes caracterizada pela própria instabilidade surgida pela incerteza quanto ao comportamento futuro do parceiro e pela aplicação da autoridade visando assegurar o comprometimento. O nível elevado de autoridade está relacionado com a estrutura de governança, adotada para monitorar, fiscalizar o relacionamento e os custos de transação estabelecidos (Williamson, 1979; Gary \& Spencer, 2000; Arbage, 2004).

As organizações podem desenvolver diversos mecanismos de alianças estratégicas, conforme explicam Motta \& Vasconcelos (2015, p. 381):

[...] estratégias como terceirização de bens e serviços, alianças estratégicas, fusões e aquisições, entre outros diferentes tipos de contratos, implicam diferentes tipos de custos de transação, fornecendo também controles maiores ou menores de uma organização sobre outra.

Os pressupostos compartilhados pelas duas correntes teóricas trazem uma grande contribuição de análise da prática organizacional. As duas abordagens convergem quando reconhecem os efeitos das relações interorganizacionais na performance das organizações.

Apesar da convergência entre os dois construtos teóricos, quando a teoria dos custos de transação é analisada de modo isolado de outras correntes teóricas, produz, assim, uma visão subsocializada dos fenômenos do campo organizacional. Na teoria dos custos de transação, a não consideração dos fatores sociais e das estruturas sociais dos processos da cadeia produtiva, distribuição e consumo organizacionais cria um modelo altamente abstrato e simplificado da realidade. Porém essa abordagem teórica apresenta um conjunto de atributos, o qual justifica a compreensão dos fenômenos do campo organizacional (Lopes, 2004).

Enquanto a teoria da dependência de recursos dá ênfase nas decisões e nas ações organizacionais e no monitoramento constante do ambiente (Bucelli et al., 2014), a teoria dos custos de transação tem como pressupostos as relações de troca entre as organizações e os demais agentes do ambiente (Bataglia et al., 2009).

Assim, a teoria dos custos de transação vê as organizações como uma composição de governança, considerando o mecanismo de minimização dos custos de transação. Portanto, a construção de estruturas organizacionais que busquem minimizar as implicações da racionalidade limitada e resguardar as transações contra o oportunismo por parte dos diversos agentes envolvidos são cruciais para as organizações (Dolci \& Maçada, 2011).

Em contrapartida, a teoria da dependência de recursos busca compreender o relacionamento das organizações e a dependência entre as instituições de forma recíproca. A dependência surge quando os gestores percebem que as implicações de um determinando ator externo impõem restrições ao desempenho e eficiência da sua organização, ou seja, a dependência passa a ser desaconselhável, pois ameaça a estabilidade e existência da organização (Gueraldi, 2006; Veiga et al., 2014).

Outro aspecto relevante de destaque da teoria da dependência de recursos é o processo por meio do qual as organizações buscam reduzir a relação de dependência do ambiente, utilizando diversas estratégias e visando assim, aumentar seu poder dentro do sistema (Balestrin \& Vargas, 2002). Nesse sentido, Veiga et al. (2014) destacam que as estratégias utilizada pelas organizações para minimizar a interdependência e as incertezas do ambiente são: fusões, aquisição, cooperação entre as empresas, troca de recursos ou troca de experiência profissional entre as organizações.

Os teóricos da teoria da dependência de recursos adotam uma visão não determinista, considerando que os gestores podem manipular o ambiente com o intuito de catalisar os recursos dos quais as organizações necessitam.

Contudo, a teoria econômica dos custos de transação está alicerçada em dois pilares: suposições comportamentais (racionalidade limitada, contratos incompletos e oportunismo) e nas características básicas que sustentam as transações (especificidade dos ativos). Nesse sentido, a teoria dos custos de transação busca compreender a dúvida entre internalizar ou terceirizar determinados meios de produção considerando três fatores: a incerteza e dificuldade de mensurar a transação; o nível de frequência com que ocorrem essas transações; e a especificidade dos bens ou serviços transacionados (Kato \& Margarido, 2000; Gueraldi, 2006).

Outro aspecto relevante da economia dos custos de transação é que a prevalência das organizações não está relacionada ao acúmulo e exploração do poder de mercado, mas sim à construção de instrumentos de mercados que visem minimizar os custos de transação (Astley \& Van de Ven, 2005). 
A teoria da dependência de recursos dá ênfase à habilidade de as organizações manterem a discrição e autonomia sobre as contingências que surgem ao longo do processo e na prerrogativa de controlar e manipular o ambiente de acordo com os objetivos propostos pelas organizações (Rossetto \& Rossetto, 2005).

Os teóricos da teoria dos custos de transação dão ênfase às transações econômica que as organizações operam, descartando o contexto social e a influência desses fatores no desempenho das empresas e nas relações de trocas.

As duas correntes teóricas são divergentes em virtude do seu foco de análise; enquanto a perspectiva da dependência de recursos reconhece os efeitos do ambiente na performance organizacional, a teoria dos custos de transação tem como base de análise as transações econômicas realizada pelas organizações.

A perspectiva da dependência de recursos também chama atenção para a capacidade de o ambiente oferecer os recursos dos quais as organizações necessitam, ou seja, seu principal enfoque é na capacidade de fornecimento de recursos escassos do ambiente. Essa interdependência das organizações com o ambiente no qual atuam concedem ao fator ambiental um papel decisivo para as empresas. Tal interdependência pode variar de acordo com a disponibilidade de recursos para as organizações (Pfeffer, 1972; Andrade \& Amboni, 2011; Thomazine \& Bispo, 2014).

Entretanto, a teoria dos custos de transação ressalta que compete aos agentes econômicos a escolha da estrutura adequada de mercados (mercado ou hierarquia), devendo esses levarem em consideração a minimização das dificuldades transacionais geradas pela racionalidade limitada e o oportunismo dos agentes envolvidos na transação (Cardenas \& Lopes, 2006).

A ideia de que existem custos para gerenciar as transações econômicas do mercado e de que o principal desafio das organizações é minimizar os custos transacionados nas trocas de recursos no meio ambiente e com outras organizações, economizando assim, tempo e recursos, constitui um dos pressupostos da teoria dos custos de transação (Souza, 2011).

Os teóricos da dependência de recursos postulam que a premência quanto às aquisições de recursos por parte das organizações e o caráter essencial da atividade desse processo organizacional tendem a ser minimizados quando este se torna irrelevante para os objetivos organizacionais (Cunha, 1993).

A sobrevivência e prosperidade das organizações estão diretamente alicerçadas ao controle que essas exercem sobre os recursos dos quais necessitam para desenvolver suas atividades (Medeiros \& Paiva, 2012). Assim, a perspectiva da dependência de recursos diverge da teoria dos custos de transação quando não considera os custos envolvidos numa operação de mercado.

Apesar do exposto acima, observa-se que a relação entre a perspectiva da dependência dos recursos e custos de transação se estabelece no melhor caminho para possível explicação do dinamismo das relações interorganizacionais.

Ao mesmo tempo em que a teoria da dependência de recursos aponta convergências e divergências com a perspectiva dos custos de transação, tendo como análise construtos teóricos diferentes, nota-se que os teóricos de cada uma tendem a mover-se juntos quando reconhecem as relações interorganizacionais.

A perspectiva da dependência de recursos surge da corrente teórica voluntarista, ou seja, as organizações têm autonomia para fazer as próprias escolhas; enquanto a perspectiva dos custos de transação nasce de uma visão determinista, logo é o ambiente que determina como as organizações devem agir em determinada circunstância.

Em síntese, as duas perspectivas proporcionam uma visão convergente para a compreensão do dinamismo que envolve as relações interorganizacionais. Motta \& Vasconcelos (2015) afirmam que as duas correntes teóricas privilegiam o grupo organizacional como base de análise e que esses pressupostos compartilhados devem ser estudados para o total entendimento das relações organizacionais.

\section{Conclusões}

Estudos sobre processos de relações interorganizacionais têm se solidificado cada vez mais nos estudos organizacionais. Contudo, cabe ainda ressaltar que o isolamento das teorias para analisar a interdependência entre as organizações não consegue explicações consistentes em sua totalidade.

O ensaio teórico revisou as suposições da teoria da dependência de recursos e custos de transação, identificando o potencial de convergência entre as abordagens, fato esse que enriquece ainda mais os estudos já construídos sobre os processos de relações interorganizacionais. Apesar da visão divergente entre as duas abordagens, seu fio condutor de convergência é identificado quando elas reconhecem a concepção das relações interorganizacionais como prerrogativa fundamental para seu funcionamento.

Diante do exposto, quando se buscam visões diferenciadas sobre a construção das relações interorganizacionais, cria-se a possibilidade da geração de diversos cenários de possíveis explicações das relações entre as organizações, fato que raramente seria admissível quando se usa uma única perspectiva como base de análise.

Há de serem consideradas, ainda, as constantes mudanças ocorridas nos últimos anos no mercado, impondo cada vez mais que as organizações desenvolvam estratégias consistentes para atender 
as demandas do mercado. Portanto, com o intuito de adaptar-se às contingências externas, as organizações muita vezes relacionam-se com outras organizações para minimizar as incertezas do futuro.

Assim, as duas perspectivas devem ser consideradas nas análises dos processos de desenvolvimento de relações organizacionais, avaliando o alcance que cada uma delas oferece para explicar as diferentes formas de relações e interdependência organizacional.

\section{Referências}

Abbade, E. B. (2010). Aplicação da teoria dos jogos na análise de alianças estratégicas. Gestão da Produção, Operações e Sistema, 5(3), 131-147.

Andrade, R., \& Amboni, N. (2011). Teoria geral da administração (2. ed.). Rio de Janeiro: Elsevier.

Arbage, A. P. (2004). Custos de transação e seu impacto na formação e gestão da cadeia de suprimentos: um estudo de caso em estruturas de governança híbridas do sistema agroalimentar no Rio Grande do Sul (Tese de doutorado). Programa de Pós-graduação em Administração, Universidade Federal do Rio Grande do Sul, Porto Alegre.

Astley, W. G., \& Van de Ven, A. H. (2005). Debates e perspectivas centrais na teoria das organizações. Revista de Administração de Empresas, 45(2), 52-73.

Balestrin, A., \& Vargas, L. M. (2002). Evidencias teóricas para compreensão das redes interorganizacionais. In Anais do $2^{\circ}$ Encontro de Estudos Organizacionais. Recife: Observatório da Realidade Organizacional, PROPAD/UFPE, ANPAD.

Balsini, C. P. V., Silveira, R. B., \& Rambo, J. (2005). Desafios do terceiro setor: uma análise baseada na perspectiva institucional, dependência de recursos e ecologia das populações. In Anais do VIII Seminários em Administração. São Paulo: USP.

Bataglia, W., Franklin, M. A., Caldeira, A., \& Silva, A. A. (2009). Implicações das teorias ambientais para administração estratégica. Revista Eletrônica de Gestão Organizacional, 7(3), 314-330.

Bucelli, D. O., Costa, P. L. O., No., Vendrametto, O. (2014). As empresas mais inovadoras: uma análise sob a ótica das teorias organizacionais. Revista GEINTEC, 4(2), 940-954.

Camilo, S. P. O., Marcon, R., \& Bandeira-de-Mello, R. (2012). Conexões políticas das firmas e seus efeitos na performance: uma convergência entre as perspectivas da governança e da dependência de recursos: um ensaio teórico. Revista Alcance Eletrônica, 19(2), 241-258.

Cardenas, L. Q., \& Lopes, F. D. (2006). A formação de alianças estratégicas: uma análise teórica a partir da teoria da dependência de recursos e da teoria dos custos de transação. Cadernos EBAPE, 4(2), 1-8. http://dx.doi. org/10.1590/S1679-39512006000200005.
Cavalcanti, A. C. L., Azevedo, L. C. S., \& Pinheiro, M. M. A. (2002). O impacto dos custos de transação sobre a terceirizações governamentais: enfoque teórico. In Anais do IX Congresso Brasileiro de Custos. São Paulo.

Costa, A. R. (2009). Inserção de fornecedores locais como fonte de desempenho em custos de transação e legitimidade: estudo de caso da cadeia produtiva de petróleo e gás do Rio Grande do Norte (Dissertação de mestrado). Centro de Ciências Sociais Aplicada, Universidade Federal do Rio Grande do Norte, Natal.

Cunha, C. F., Saes, M. S. M., \& Mainville, D. Y. (2015). Custos de transação e mensuração na escolha da estrutura de governança entre supermercados e produtores agrícolas convencionais e orgânicos no Brasil e nos EUA. Gestão \& Produção, 22(1), 67-81. http://dx.doi. org/10.1590/0104-530X046-12.

Cunha, M. P. (1993). Organizações, recursos e a luta pela sobrevivência: análise aos níveis organizacionais e ecológicos. Revista de Administração de Empresas, 33(5), 34-47.

Dimaggio, P. J., \& Powell, W. W. (2005). A gaiola de ferro revisitada: isomorfismo institucional e racionalidade coletiva nos campos organizacionais. Revista de Administração de Empresas, 45(2), 74-89.

Diniz, B. A., \& Marconatto, D. A. (2011). Confiança: um construto universal. Revista de Administração FACES Journal, 10(1), 35-55.

Dolci, P. C., \& Maçada, A. C. G. (2011). Um modelo conceitual de governança da cadeia de suprimentos: analisando suas concepções e elementos sob a ótica das teorias da governança. In Anais do XXXV EnANPAD. Rio de Janeiro: ANPAD.

Gary, S., \& Spencer, D. A. (2000). The uncertain foundations of transaction costs economics. Journal of Economic Issues, 34(1), 61-87. http://dx.doi.org/10.1080/00213 624.2000 .11506244 .

Gonçalves, W. M. (2008). Confiança, ECT e formas organizacionais: um framewok aplicado na vitinicultura da Serra Gaúcha e do vale do São Francisco (Tese de doutorado). Programa de Pós-graduação em Agronegócio, Universidade Federal do Rio Grande do Sul, Porto Alegre.

Gueraldi, R. G. (2006). A interface entre os setores público e privado: uma perspectiva estratégica. In Anais do EnANPAD. Salvador: ANPAD.

Ilha, V. T. (2010). A teoria dos custos de transação e o modelo de comercialização de energia elétrica após as reformas do setor elétrico (Monografia). Departamento de Ciências Econômicas, Universidade Federal do Rio Grande do Sul, Porto Alegre.

Jacobs, D. (1974). Dependency and vulnerability: na exchange approach to the control of organizations. Administrative Science Quarterly, 19(1), 45-59. http:// dx.doi.org/10.2307/2391787. 
Kato, H. T., \& Margarido, M. A. (2000). Economia dos custos de transação (ECT): análise do conflito das bananas. Revista de Administração de Empresas, 35(4), 13-21.

Lopes, F. D. (2004). Perspectiva teórica contemporânea nos estudos das organizações: apresentação de um modelo de análise da formação de joint venture internacionais. Cadernos EBAPE, 2(3), 1-21. http://dx.doi.org/10.1590/ S1679-39512004000300005.

Medeiros, L. C., \& Paiva, P. A. (2012). Ecologia populacional e escolhas estratégicas: uma proposta de convergência. In Anais do XXXVI EnANPAD. Rio de Janeiro: ANPAD,.

Morgan, G. (2002). Imagens da organização: edição executiva (2. ed.). São Paulo: Atlas. 375 p.

Motta, F. C. P., \& Vasconcelos, I. F. G. (2015). Teoria geral da administração (3. ed.). São Paulo: Cengage Learning.

Pereira, A. J., Dathein, R., \& Conceição, O. A. C. (2014). A empresa e seu ambiente de interação: os limites da Teoria dos Custos de Transação e o alcance da Teoria Institucionalista Evolucionária. Economia e Sociedade, Campinas, 23(1), 33-61. http://dx.doi.org/10.1590/ S0104-06182014000100002.

Pfeffer, J. (1972). Merger as a response to organizational interdependence. Administrative Science Quarterly, 17(3), 382-394. http://dx.doi.org/10.2307/2392151.

Puffal, D. P., \& Puffal, C. W. (2014). A evolução do campo de estudos em redes interorganizacionais: uma análise de publicações internacionais das relações entre empresas. Revista Brasileira de Gestão e Inovação, 1(3), 63-86.

Rocha, J. S., et al (2011). Teoria da dependência de recursos explicando a percepção de governança corporativa de importantes tomadores de decisão. R. Cont. Ufba, 5(3), 31-44.

Rossetto, C. R., \& Rossetto, A. M. (1999). A combinação das perspectivas institucionais e da dependência de recursos no estudo de adaptação estratégica organizacional. Teoria e Evidência Econômica, 7(12), 111-136.

Rossetto, C. R., \& Rossetto, A. M. (2005). Teoria institucional e dependência de recursos na adaptação organizacional: uma visão complementar. Revista de Administração de Empresas Eletrônica, 4(1), 1.

Sacomano, M., No., \& Truzzi, O. M. S. (2002). Perspectivas contemporâneas em análise organizacional. Gestão \& Produção, 9(1), 32-44. http://dx.doi.org/10.1590/ S0104-530X2002000100004.
Santos, M. S., Stamm, C., \& Shikida, P. F. A. (2014). Inovação, economia dos custos de transação e gestão da cadeia de suprimento: uma discussão teórica. Revista Politica e Planejamento Regional, 1(2), 257-272.

Schlabitz, C. J. (2008). Custo de transação e o mercado de crédito brasileiro: uma análise sob a ótica da nova economia institucional (Dissertação de mestrado). Programa de Pós-graduação em Economia, Universidade Federal do Rio Grande do Sul, Porto Alegre.

Schubert, M. N. (2012). Análise dos custos de transação nas cooperativas de produção de leite no oeste de Santa Catarina. (Dissertação de mestrado). Programa de Pósgraduação em Desenvolvimento Rural, Universidade Federal do Rio Grande do Sul, Porto Alegre.

Silva, E. B., Fo. (2006). A economia dos custos de transação e a abordagem da competências: elementos para teoria institucionalista unificada da firma (Dissertação de mestrado). Programa de Pós-graduação em Economia, Universidade Federal do Rio Grande do Sul, Porto Alegre.

Souza, R. K. S. (2011). Sobre (vivência) e mortalidade de pequenos negócios: o caso de mercados varejistas potiguares (Dissertação de mestrado). Centro de Ciências Sociais Aplicada, Universidade Federal do Rio Grande do Norte, Natal.

Thomazine, J. S., \& Bispo, F. C. S. (2014). Dependência de recursos e custos de transação: uma análise sobre os motivos da terceirização. In Anais do XI Simpósio de Excelência em Gestão e Tecnologia. SEGeT.

Trevisan, M. (2013). A ecologia industrial e as teorias de sistemas, institucional e da dependência de recursos a partir dos atores de um parque tecnológico (Tese de doutorado). Programa de Pós-graduação em Administração, Universidade Federal do Rio Grande do Sul, Porto Alegre.

Veiga, C. P., Veiga, C. R. P., \& Catapan, A. (2014). Análise da alteração de canais de distribuição pela visão integrada da teoria institucional e dependência de recursos. In Anais do SIMPOI. SIMPOI.

Viana, F. L. E., \& Añez, M. E. M. (2008). Contribuições teóricas dos estudos organizacionais para a dinâmica da gestão da cadeia de suprimentos. Interface, 5(1), 57-74.

Williamson, O. E. (1979). Transaction-cost economics: the governance of contractual relations. The Journal of Law \& Economics, 22(2), 233-261. http://dx.doi. org/10.1086/466942. 